\title{
LAS PARADOJAS MATEMÁTICAS: UNA INTRODUCCIÓN PARA ESTUDIANTES Y MAESTROS DE EDUCACIÓN MEDIA
}

Rolando Sáenz, Andrade

Universidad Central del Ecuador

Recibido: 12 - septiembre - 2015, aprobado: 10 - noviembre - 2015

\section{Resumen}

En este trabajo nos proponemos presentar paradojas de una manera sencilla para que sean accesibles a los estudiantes y profesores de la escuela secundaria y los estudiantes que comienzan la universidad. El objetivo es motivar a los jóvenes a pensar con lógica para introducirlos en la forma de pensar en las matemáticas. Se consideran tres tipos de paradojas: paradojas que no son paradojas reales, paradojas semánticas y paradojas lógicas.

Palabras clave: paradoja, teoría matemática, teoría de conjuntos, lógica, matemáticas, semántica.

\begin{abstract}
In this work we intend to present paradoxes in a simple way, in order to make them accessible to high school students, teachers and to people that begin their university studies. The aim is to motivate young people to think with logic and to introduce them in the mathematical way of thinking. We consider three types of paradoxes: paradoxes that are not real paradoxes, semantic paradoxes and logic paradoxes.
\end{abstract}

Keywords: paradox, mathematical theory, set theory, logic, mathematics, semantics. 


\section{1. ¿Qué es una Paradoja?}

No es nuestra intención dar una definición precisa de paradoja en términos filosóficos ni de introducir sus diferentes clases, sino más bien presentarla en un sentido amplio, en la forma que es comprendida dentro del ámbito matemático y, más aún, por la mayoría de la gente corriente. En ese sentido entenderemos por paradoja por lo menos en un principio todo razonamiento matemático contrario a la intuición y a la opinión general o toda expresión o idea matemática que involucra una incompatibilidad aparente opuesta a lo que habitualmente se considera verdadera.

Desde este punto de vista podemos clasificar a las paradojas matemáticas en tres categorías:

- Aquellas que son consecuencias de razonamientos falsos (falacias).

- Resultados extraños que aunque van más allá de la intuición, son verdaderos.

- Paradojas lógicas que constituyen verdaderas contradicciones.

\section{Algunas paradojas clásicas}

Tal vez la mejor manera de familiarizarnos con las paradojas que se presentan en matemáticas es analizar con algún detenimiento algunas de ellas. Para ello, las hemos escogido de entre aquellas las más conocidas, ante las cuales la mayoría de nosotros hemos tenido la oportunidad de asombrarnos cuando nos la presentó el maestro en la escuela, dejándonos admirados con su sapiencia, o la leímos en algún libro de juegos matemáticos o en una conocida obra maestra de la literatura universal.

He aquí algunas de ellas:

\section{A. La prueba del nueve (Tomado de "La magie des paradoxes").}

Jacinto: Samanta, piensa en un número cualquiera

Samanta piensa en el número 52389.

Jacinto: reordena las cifras del número que pensaste de tal manera que obtengas otro número.

Samanta: selecciona el número 28593 que tiene las mismas cifras que el número original.

Jacinto: Resta el número mayor menos el menor y escoge una cifra cualquiera distinta de cero del resultado.

Samanta: escoge el 3 de la diferencia $23796=52389-28593$.

Jacinto: Dime las cifras restantes en el orden que desees.

Samanta: $7-9-6-2$.

Jacinto: suma mentalmente estas cifras y el resultado 24 lo divide por 9 obteniendo como residuo 6. La cifra seleccionada por Samanta es $9-6=3$, resultado que anuncia ante el asombro de Samanta. 
Lo que parece un acto de magia y contrario al sentido común tiene una explicación sencilla y es la base de la conocida prueba del 9 para las cuatro operaciones elementales:

El residuo de la división de un número entero por 8 es igual al residuo de la división de la suma de sus cifras por 9. Luego, si $a$ y $b$ tienen las mismas cifras, las divisiones de estos números por 9 tienen el mismo residuo y su diferencia a-b es múltiplo de 9. Para hallar la cifra escondida bastará entonces añadir a la suma de las cifras enunciadas el número que falta para obtener un múltiplo de $9(24+3)$ o, lo que es lo mismo, añadir al residuo (6) el número que falta para obtener 9 (3).

Observe que una forma simple de obtener el residuo es quitar 9 a la suma de las cifras cada vez que ésta es superior o igual a 9:

$$
\begin{aligned}
& 7+9=16,16-9=7 \text {, } \\
& 7+6=13,13-9=4,4+2=6 \text {. }
\end{aligned}
$$

Si a un hilo que rodea la Tierra por el Ecuador de tal manera que esté ceñido a ella, aumentamos su longitud en un metro. ¿es perceptible la distancia que éste se separa del suelo? Suponemos que la Tierra es lisa como una pelota, sin accidentes geográficos.

Realicemos los cálculos para lo cual, por sencillez y sin que esto deforme nuestros cálculos, aproximaremos el radio de la Tierra a $6000 \mathrm{~km}$ (en lugar de 6370) o $6 \times 106$ metros.

La longitud del hilo es originalmente igual a la circunferencia de la Tierra por el Ecuador:

$2 \pi(6 \times 106)=37699111,84 \mathrm{~m}$.

$\mathrm{Al}$ añadir 1 m obtenemos una circunferencia de 37699112,84 metros que coresponde a un radio de

$37699911,84 / 2 \pi=6000000,16 \mathrm{~m}$.

La diferencia entre los dos radios es dieciséis centésimas de metro, 16 centímetros, ¡totalmente perceptible! Este resultado aparentemente paradójico, que choca con nuestra intuición que anunciaba que el hilo hubiera permanecido ceñido al suelo, se debe posiblemente a que con grandes números perdemos el sentido de la proporción, y que $16 \mathrm{~cm}$ con respecto a 6 millones de metros es "imperceptible".

\section{C. "Preferir a" no es una relación transitiva.}

En una encuesta electoral en la que participan tres candidatos A, B y C; $2 / 3$ de los encuestados prefieren $\mathrm{A}$ a $\mathrm{B}$ e, igualmente, $2 / 3$ de los encuestados prefieren $\mathrm{B}$ a C. ¿Podemos concluir que A tiene las mejores posibilidades de ganar las elecciones?

Para simplificar el razonamiento consideremos una muestra de nueve personas encuestadas, divididas en tres grupos G1, G2 y G3 de tres personas cada uno. 
Los del primer grupo manifestaron preferir $A$ a $B$ y $B$ a $C$, los del segundo $B$ a $C$ y $C$ a $A$, y los del tercer grupo expresaron su preferencia por $A$ con relación a $B$ y por $C$ con relación a $A$. Se tiene así que seis personas -los $2 / 3$ de los encuestados- prefieren $C$ a $A$.

El siguiente grupo de paradojas es esencialmente diferente al anterior.

\section{La paradoja del mentiroso}

Esta paradoja se atribuye al cretense Epiménides que se supone vivió en el siglo VI antes de nuestra era.

Los cretenses son mentirosos dice Epiménides.

Si Epiménides dice la verdad, él, por ser cretense, es mentiroso y por tanto su afirmación es falsa. Si por el contrario Epiménides miente, los cretenses no son mentirosos y él, como cretense, dice la verdad.

Si Epiménides dice la verdad entonces miente

Si Epiménides miente entonces dice la verdad

\section{La paradoja del barbero}

Esta paradoja fue propuesta por Bertrand Russell.

Yo afeito a todos los hombres de Sevilla que no se afeitan a sí mismos y únicamente a ellos, dice el barbero de Sevilla.

¿Quién afeita al barbero de Sevilla?

Si se afeita él mismo, es uno de los hombres que se afeitan a sí mismos y por tanto no puede afeitarse él mismo.

Si le afeita otra persona, de acuerdo con la declaración del barbero, esa persona debe ser el mismo.

Nadie afeita al barbero

\section{La paradoja de Sancho Panza}

En "El Quijote” de Cervantes se encuentra al siguiente pasaje (que no lo expreso en forma textual):

En una cierta isla, de la cual Sancho Panza se convirtió en soberano, los visitantes debían contestar la pregunta: ¿A qué viene usted? Si el visitante decía la verdad era fusilado y si mentía era colgado. Un día, un viajero llegó a la isla y respondió a la pregunta: "vengo para ser colgado".

Si ahorcaban al viajero, éste había dicho la verdad y debía ser fusilado. Por el contrario, si lo fusilaban, había mentido y debía ser colgado. Ante la imposibilidad de hacer cumplir la ley, el gobernador dejó libre al viajero.

\section{La paradoja de Berry}

La paradoja que presentamos es una adaptación al castellano de la paradoja original de Berry. 
Sea $A$ "el conjunto de todos los números naturales que pueden describirse con menos de veinte palabras del idioma castellano".

Puesto que el número de palabras del idioma castellano es finito, el conjunto $A$ es finito y existen números naturales que son más grandes que todos los elementos de $\mathrm{A}$ y que por tanto no pertenecen a $A$. Tomemos el más pequeño de estos números: "el más pequeño número natural que no puede describirse con menos de veinte palabras del idioma castellano". La frase entre comillas tiene diecisiete palabras y describe precisamente a ese número en menos de veinte palabras y, en consecuencia, pertenece al conjunto $A$.

Antes de realizar un análisis un poco más detenido de las paradojas y presentar el significado que tuvieron, y aún lo tienen, en la fundamentación misma de las matemáticas, vía una explicación previa, aunque breve de esos fundamentos, es conveniente que hagamos hincapié en ciertos aspectos que se presentan en los ejemplos con los que hemos iniciado este artículo. Los tres primeros en realidad no constituyen paradojas y si los resultados que se obtienen son aparentemente paradójicos, o más bien parecen chocar con el sentido común, es porque presentan situaciones con las que no estamos familiarizados, que se fundamentan en resultados matemáticos, aunque generalmente simples, poco usuales -criterios de divisibilidad especiales, relaciones no transitivas- o se refieren a cantidades muy grandes o muy pequeñas que normalmente no se presentan en nuestra vida cotidiana.

En cualquier caso, estas pequeñas "paradojas" no resisten un análisis detenido y constituyen simples juegos, a menudo interesantes, que recrean nuestro intelecto y nos obligan a pensar pero que ningún momento ponen en peligro la consistencia de las matemáticas.

Los cuatro últimos ejemplos constituyen auténticas paradojas que en su momento sacudieron las bases mismas de la teoría de conjuntos de Cantor y obligaron a revisar la concepción misma de conjunto a fin de evitar la formación de conjuntos definidos por condiciones que no son aceptables en una teoría matemática: "el conjunto de los números naturales que pueden describirse con menos de veinte palabras del idioma castellano"; o de conjuntos que incluyen "demasiado": en la paradoja del barbero un elemento del conjunto (el barbero) está en relación $\mathrm{R}$ (afeitar a) con todos los elementos que no están en relación $\mathrm{R}$ con sí mismos (los barberos que no se afeitan a sí mismos).

Para comprender mejor estas paradojas, sus implicaciones y las soluciones propuestas, es conveniente que digamos antes unas pocas palabras sobre lógica y teoría de conjuntos.

\section{Algo de lógica y conjuntos}

Siguiendo la "Teoría de Conjuntos" de Bourbaky, una teoría matemática está conformada por:

1. Los signos lógicos $((\neg, \bigvee, \wedge, \Rightarrow \Leftrightarrow))$, letras y signos de la Teoría (por ejemplo " $\in$ " en la teoría de conjuntos). 
2. Los ensamblajes que son sucesiones finitas de signos que, de acuerdo con ciertas reglas, se clasifican en términos y relaciones. He aquí algunos ejemplos:

- Las letras son términos

- $\quad$ Los siguientes ensamblajes son relaciones: $\neg$ p,p $\Rightarrow q, p \vee q$.

3. Los axiomas son relaciones explícitas o implícitas (se deducen a partir de ciertos esquemas o reglas de formación) que se fijan de antemano.

4. Los teoremas o relaciones verdaderas son cualquiera de las relaciones que aparecen en una demostración. Una demostración es una sucesión de relaciones $\mathrm{R}$ donde $\mathrm{R}$ es un axioma o está precedida por relaciones de la forma $\mathrm{S}$ y $\mathrm{S} \Rightarrow \mathrm{R}$ (regla de inferencia). En particular, un axioma es un teorema.

Una relación $\mathrm{R}$ es falsa si su negación $\neg \mathrm{R}$ es verdadera. Una teoría es contradictoria si existe una relación que es a la vez verdadera y falsa.

Una teoría matemática dotada de suficientes axiomas, de tal manera que sean teoremas todas las "tautologías" que se obtienen usando las tablas de verdad, se llama una teoría lógica. Los siguientes esquemas de formación de axiomas satisfacen esta condición:

Cualesquiera que sean las relaciones $\mathrm{A}, \mathrm{B}$ y $\mathrm{C}$, las relaciones siguientes son verdaderas:

L1. $A \vee A \Rightarrow A$.

L2. $A \Rightarrow A \vee B$.

L3. $A \vee B \Rightarrow B \vee A$.

L4. $(A \Rightarrow B) \Rightarrow((C \vee A) \Rightarrow(C \vee B))$.

Una teoría cuantificada es una teoría lógica con un nuevo signo (B) y un criterio adicional de formación de relaciones: "Si x es una letra y $\mathrm{R}$ una relación, ( $\mathrm{Gx}$ ) R ("existe un $\mathrm{x}$ tal que R") es una relación. Se añade además un esquema de formación de axiomas que intuitivamente expresa que si $\mathrm{R}$ es una relación que expresa una propiedad sobre $\mathrm{x}, \mathrm{y}$ al reemplazar $\mathrm{x}$ por un objeto $\mathrm{T}$ se obtiene una relación verdadera, entonces ( $(9 \mathrm{x}) \mathrm{R}$ es una relación verdadera.

Finalmente llegamos a la teoría de conjuntos. La presentación que hacemos aquí en forma simplificada, es una de las muchas formas que existen de presentar la teoría de conjuntos, sin embargo, la mayoría de ellas conducen a los mismos resultados.

La teoría de conjuntos es una teoría cuantificada en la que se ha introducido el símbolo específico $\in$ y el criterio de formación de relaciones:

Si a y A son términos, a $\in A$, que se lee "a pertenece a A", es una relación.

A los términos de esta teoría los llamaremos conjuntos. La negación de la relación $a \in A$ la notaremos a $a \notin A$ (a no pertenece a $A$ ).

Si $A$ y $B$ son conjuntos $A \subset B$ es la relación " $x \in A \Rightarrow x \in B$ ", $y A=B$ es la relación

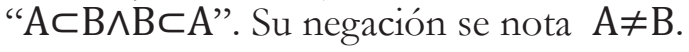

La teoría de conjuntos tiene, además de los axiomas de una teoría cuantificada, 
sus propios axiomas. Uno de ellos, el axioma de extensión, establece que si a, b y A son conjuntos

$\mathrm{a}=\mathrm{b}$ y $\mathrm{a} \in \mathrm{A}$ implica que $\mathrm{b} \in \mathrm{A}$.

Otro axioma, el axioma de construcción de conjuntos o axioma de especificación -y con esto llegamos al problema crucial que permitió la aparición de las paradojas- establece, por lo menos de acuerdo con la teoría de conjuntos de Cantor, que si $\mathrm{R}(\mathrm{x})$ es una condición sobre $\mathrm{x}$ (en realidad una relación que contiene la letra $\mathrm{x}$ ), existe el conjunto $\mathrm{C}$ de todos los elementos de $\mathrm{x}$ que se satisfacen la condición $\mathrm{R}(\mathrm{x})$.

$$
C=\{x \mid R(x)\}
$$

Este axioma es en suma útil y permite formar un sinnúmero de conjuntos como se observa en los siguientes ejemplos:

El conjunto vacío:

$\varnothing=\{\mathrm{x} \mid \mathrm{x} \neq \mathrm{x}\}$.

La unión de los conjuntos $\mathrm{A}$ y $\mathrm{B}$

$\mathrm{A} \cup \mathrm{B}=\{\mathrm{x} \mid \mathrm{x} \in \mathrm{A} \vee \mathrm{x} \in \mathrm{B}\}$

La intersección de los conjuntos $\mathrm{A}$ y $\mathrm{B}$.

$A \cap B=\{x \mid x \in A \wedge x \in B\}$.

Por supuesto, la teoría de conjuntos tiene otros axiomas que no es de nuestro interés tratarlos aquí.

\section{Paradojas lógicas y paradojas semánticas}

Dentro de este grupo de paradojas se incluyen los últimos cuatro ejemplos presentados al inicio. La paradoja del barbero de Russell es el prototipo de las paradojas lógicas y, recordemos que su característica consiste en relacionar un elemento del conjunto con todos los elementos que no están relacionados con sí mismos. Conseguimos exactamente el mismo efecto si consideramos el conjunto

$\mathrm{A}=\{\mathrm{x} \mid \mathrm{x} \notin \mathrm{x}\}$.

Nos preguntamos entonces ¿cuál de las afirmaciones $A \in A$ o $A \notin A$ es verdadera.

Si $A \in A$, por la definición misma del conjunto $A$ se sigue que $A \notin A$.

Si $A \notin A$, entonces $A$ satisface la condición que define al conjunto $A(x \notin x)$ y por tanto $A \in A$.

Concluimos que $A \in A$ si y solamente si $A \notin A$.

La similitud con la paradoja del barbero es evidente.

Puede parecer extraña una relación del tipo $\mathrm{x} \in \mathrm{x}$ o $\mathrm{x} \notin \mathrm{x}$, sin embargo están de acuerdo con las reglas de formación de relaciones. Si en algo puede ayudar a aceptar este tipo de relaciones, con las debidas reservas como veremos más adelante, pensemos en el conjunto de todos los conjuntos que son elemento de sí mismo, o en el conjunto de todos los objetos que no son libros. 
Ventajosamente, pequeños cambios en la teoría de conjuntos permiten evitar este tipo de paradojas. Uno de ellos fue propuesto por von Newmann.

Von Newmann, a los términos los llamó clases y distinguió dos tipos de clases: las que pertenecen a alguna clase, a las cuales denominó elementos o conjuntos, y aquellas que no pertenecen a ninguna clase. En su teoría, el axioma de construcción de conjuntos es un axioma de construcción de clases.

Regresemos ahora a las paradojas lógicas y sea $\mathrm{A}$ la clase $\mathrm{A}=\{\mathrm{x} \mid \mathrm{x} \notin \mathrm{x}\}$.

Si $A$ es un elemento debe tenerse que $A \in A$ o $A \notin A$. Puesto que cualquiera de estas afirmaciones nos lleva a una contradicción, la única conclusión posible es que $\mathrm{A} \in \mathrm{A}$ si y solamente si $\mathrm{A}$ no es un elemento. ¡No existe paradoja!.

Los otros ejemplos, las paradojas del mentiroso, de Sancho Panza y de Berru se clasifican dentro de las paradojas semánticas y, aunque existe una estrecha relación entre las paradojas semánticas y las paradojas lógicas -en realidad las paradojas semánticas pueden transformarse en lógicas y viceversa- notemos que las primeras tienen como patrón el hecho de que en ellas se hacen enunciados acerca del lenguaje (lenguaje objeto) en el cual se hacen enunciados sobre objetos. Son enunciados de enunciados (metalenguaje). "Todo lo que dicen los cretenses es mentira" es un enunciado sobre "lo que dicen" los cretenses. "El más pequeño número natural que no puede describirse con menos de veinte palabras del idioma castellano" es un enunciado acerca del lenguaje en el cual se codifica la aritmética. Simplemente se están mezclando dos lenguajes. La solución encontrada para evitar las paradojas semánticas es entonces "relativamente" sencilla: no hay como salirse del lenguaje establecido dentro de la teoría. La forma como hemos introducido las teorías matemáticas impide la formación de conjuntos como "el conjunto de todos los números naturales que pueden describirse con menos de veinte palabras del idioma castellano". En efecto, ¿cómo podríamos expresar este conjunto en la forma $\{\mathrm{x} \mid \mathrm{R}(\mathrm{x})\}$, si $\mathrm{R}(\mathrm{x})$ es una relación y una relación es un ensamblaje, y este a su vez es una sucesión de símbolos que pueden únicamente ser letras o signos lógicos o los signos $\exists$ o $\in$, dispuestos de acuerdo con reglas precisas.

Por cierto, los conjuntos de Cantor eran mucho más generales e intuitivos: "una colección de objetos cuyas propiedades pueden describirse". El conjunto de Berry cabe perfectamente dentro de esta concepción.

Terminamos esta sección haciendo mención a -tal vez- la más interesante de las paradojas semánticas.

\section{La paradoja de Richard (Tomado de "sigma, El mundo de las matemáticas")}

A cada propiedad de los mismos enteros positivos, descrita en un determinado lenguaje (el castellano por ejemplo), se le asigna un único entero de acuerdo con el número de letras de la frase que describe la propiedad y a un orden alfabético en caso de que el número de letras de dos frases coincida. Así por ejemplo, la propiedad de ser número par se le puede describir por "divisible por dos" y asignarle el entero 15. Diremos que un entero es "richardiano" si no tiene la propiedad a la cual está asignado. Así, en el ejemplo, 15 es richardiano pues no es "divisible por 
dos". Ahora bien la expresión "no tener la propiedad a la cual está asignado" que describe la propiedad de ser richardiano debe estar asignado a un número n. ¿Es este número $\mathrm{n}$ richardiano? El lector tiene la respuesta.

\section{Algunas preguntas y conclusiones}

La axiomática de conjuntos de Newmann no es la única forma de evitar las paradojas tipo Russell. Zermelo, por ejemplo, sin diferenciar clases y elementos, propuso el siguiente axioma de construcción de conjuntos: "Si C, es un conjunto y $\mathrm{R}(\mathrm{x})$ una condición que tiene sentido para cada elemento $\mathrm{x}$ de $\mathrm{C}$, existe el conjunto de los elementos $x$ de $C$ que satisfacen la condición $R(x):\{x \in C \mid R(x)\}$.

¿Por qué con este axioma no tienen cabida las paradojas tipo Russell?

Este axioma de Zermelo prohíbe también la existencia del conjunto universo (el conjunto de todos los conjuntos), ¿por qué sin embargo, en el sistema de von Newman si es posible hablar de la clase universo, ¿cómo?, pero ésta ciertamente no es un conjunto bajo el supuesto de que toda subclase de un conjunto es un conjunto.

Para evitar las paradojas que volverán contradictoria a la teoría de conjuntos ha sido necesario -en cualquiera de los sistemas propuestos- sacrificar algo (la existencia del conjunto universo, establecer una diferencia entre conjuntos y clases), sin embargo y a pesar de que pueden aparecer nuevas paradojas, la teoría de conjuntos es suficientemente sólida para soportar todo el edificio de las matemáticas que sobre ella se ha construido. De hecho existen paradojas para las cuales no se ha encontrado una solución satisfactoria. Una de ellas es la paradoja de Newcomb.

Se dispone de dos cajas A y B la primera es transparente y contiene dinero y la otra es opaca. La opaca contiene 1000 veces más dinero que la transparente o está vacía.

Un extraterrestre tiene la capacidad de predecir con bastante precisión las decisiones que van a tomar las personas de llenar o vaciar la caja opaca B.

El juego consiste en: 1) escoger la caja B en cuyo caso el extraterrestre, prediciendo la intención de la persona colocará el dinero en la caja B; 2) escoger las dos cajas, en cuyo caso el extraterrestre dejará vacía la caja B.

Una vez que el extraterrestre ha tomado una decisión no puede cambiarla.

Una primera persona escoge la caja B.

Una segunda persona escoge las dos cajas pues razona que cualquiera que haya sido la decisión del extraterrestre, una vez tomada, ésta no puede cambiar.

¿Cuál de las dos personas tiene la razón?

Proponemos al lector examinar las siguientes paradojas, algunas de ellas clásicas otras tomadas de diferentes autores, determinar si realmente pueden ser consideradas como tales y clasificarlas.

I. Sean a, b y c números tales que a

$(\mathrm{a}+\mathrm{b})^{\wedge} 2=(\mathrm{a}+\mathrm{b}) \mathrm{c}$ 
$a^{\wedge} 2+a b-a c=-a b-b^{\wedge} 2+b c$

$\mathrm{a}(\mathrm{a}+\mathrm{b}-\mathrm{c})=-\mathrm{b}(\mathrm{a}+\mathrm{b}-\mathrm{c})$

Como a y b son números cualesquiera se sigue, por ejemplo, que, $1=-2$.

II. La regla "toda regla tiene excepciones" ¿es verdadera o falsa?

III. ¿Por qué las frases siguientes son contradictorias?

- Solo sé que nada sé (Sócrates)

- $\quad$ Se prohíbe fijar avisos en esta pared

IV. El matemático Kurt Gulling divide a todos los adjetivos en dos conjuntos disjuntos, el de los adjetivos autodescriptivos y el de los adjetivos no - autodescriptivos. ¿A qué conjunto pertenece el adjetivo no - autodescriptivo?

V. ¿Qué se puede decir de la frase 3 en el siguiente grupo de frases?

1. París es la capital de España.

2. García Márquez escribió “El Quijote”.

3. Las frases 1,2 y 3 son falsas.

VI. La siguiente tabla representa la esperanza de vida de una cierta población:

\begin{tabular}{|l|c|c|c|c|c|c|c|c|c|c|}
\hline Edad & 5 & 10 & 15 & 20 & 25 & 30 & 35 & 40 & 45 & 50 \\
\hline Esperanza & 62 & 60 & 58 & 56 & 52 & 47 & 43 & 38 & 34 & 29 \\
\hline
\end{tabular}

Escogemos una edad cualquiera, 25 por ejemplo, y la esperanza de vida correspondiente 52. La edad más cercana a 52 es 50 y su esperanza de vida 29. Continuando con este proceso se llega a $(35,43),(45,34)$ y nuevamente a $(35,43)$. Observamos que $(40,38)$, que se encuentra entre los dos pares anteriores, corresponde precisamente a la edad en la que el tiempo vivido y el tiempo posible que resta de vida es aproximadamente el mismo. Cualquier otra edad inicial y cualquier tabla de mortalidad nos llevarán al mismo resultado.

VII. El cocodrilo dice a la madre del niño: “¿Voy a comerme a tu hijo?” Si me respondes con la verdad te devolveré a tu hijo sano y salvo.

¿Qué podría contestar la madre para salvar a su hijo?

VIII. Platón: Lo que dirá Sócrates será falso.

Sócrates: Platón ha dicho la verdad.

¿Qué se puede decir de la veracidad o falsedad de estas frases?

Roberto sale a las $8 \mathrm{~h} 00$ de A y llega a las $18 \mathrm{~h} 00$ a B. Al día siguiente sale de B a las 8 h00 y llega a A a las $18 \mathrm{~h} 00$ por el mismo camino que recorrió el día anterior, pero la primera mitad del camino lo recorre más lentamente que a la ida.

¿Existe un punto del recorrido por el cual Roberto pasa exactamente a la misma hora tanto a la ida como al regreso? 


\section{Bibliografía}

Bourbaki, N. (s/f). Eléments de mathématique. Paris: Hermann.

Gardner, M. (1980). La Magie de Paradoxes. Paris: Pour La Sciencie.

Halmos, P. (1960). Naive Set Theory. New York: Van Nostrand Campany.

Muñoz, J. (1969). El mundo de las matemáticas. Barcelona: Ediciones Grijalbo.

Pinter, C. (1971). Set theory. Reading-Massachussetts: Addison-Wesley. 